\title{
Ocneanu's trace and Starkey's rule
}

\author{
Meinolf Geck and Nicolas Jacon
}

\begin{abstract}
We give a new simple proof for the weights of Ocneanu's trace on Iwahori-Hecke algebras of type $A$. This trace is used in the construction of the HOMFLYPT-polynomial of knots and links (which includes the famous Jones polynomial as a special case). Our main tool is Starkey's rule concerning the character tables of Iwahori-Hecke algebras of type $A$.
\end{abstract}

\section{Introduction}

Let $q$ be an indeterminate and $K=\mathbb{Q}(q)$ the field of rational functions in $q$. Let $\mathcal{H}_{n}(q)$ be the Iwahori-Hecke algebra associated with the symmetric group $\mathfrak{S}_{n}$ (of type $\left.A_{n-1}\right)$. Thus, $\mathcal{H}_{n}(q)$ is an associative $K$-algebra with a basis $\left\{T_{w} \mid w \in \mathfrak{S}_{n}\right\}$ and the multiplication is determined by the following rules.

$$
\begin{aligned}
T_{s_{i}}^{2} & =q T_{1}+(q-1) T_{s_{i}} & & \text { for } 1 \leqslant i \leqslant n-1, \\
T_{w} T_{w^{\prime}} & =T_{w w^{\prime}} & & \text { if } l\left(w w^{\prime}\right)=l(w)+l\left(w^{\prime}\right) .
\end{aligned}
$$

Here, $s_{i}$ denotes the basic transposition $(i, i+1)$ and $l(w)$ denotes the length of a shortest possible expression of $w$ as a product of the transpositions $s_{i}$. (It can be shown that $l(w)$ is the number of inversions of the permutation $w \in \mathfrak{S}_{n}$, that is, the number of pairs $i<j$ such that $w(i)>w(j)$.) See also the description of $\mathcal{H}_{n}(q)$ given by Jones $[9, \S 4]$. (We have $T_{s_{i}}=g_{i}$ in Jones' notation.)

A trace function is a $K$-linear map $\psi: \mathcal{H}_{n}(q) \rightarrow K$ such that $\psi\left(h h^{\prime}\right)=\psi\left(h^{\prime} h\right)$ for all $h, h^{\prime} \in \mathcal{H}_{n}(q)$. Now let $z \in K$. A trace function $\tau: \mathcal{H}_{n}(q) \rightarrow K$ with $\tau\left(T_{1}\right)=1$ is called an Ocneanu trace with parameter $z$ if $\tau\left(T_{w} T_{s_{m}}\right)=z \tau\left(T_{w}\right)$ for any $1 \leqslant m \leqslant n$ and any $w \in\left\langle s_{1}, \ldots, s_{m-1}\right\rangle$. By [9, Theorem 5.1], for any $z \in K$, there exists a unique Ocneanu trace $\tau$ with parameter $z$; we will therefore write $\tau=\tau_{z}$. (See also $[6, \S 4]$ for an alternative construction of $\tau_{z}$.)

Another characterisation of Ocneanu's trace (which will be used later in this paper) can be given as follows. Every trace function is uniquely determined by its values on basis elements $T_{w}$ where $w$ runs over a certain set of representatives of the various conjugacy classes of $\mathfrak{S}_{n}$ (see [7, 8.2.6]). Following [7, 3.1.16], these particular representatives can be described as follows. The conjugacy classes of $\mathfrak{S}_{n}$ are naturally parametrised by the partitions $\mu \vdash n$. If $\mu$ has non-zero parts $\mu_{1}, \mu_{2}, \ldots$, then we take $w_{\mu}:=s_{i_{1}} s_{i_{2}} \cdots s_{i_{k}}$ as representative in the class labelled by $\mu$, where $\left\{i_{1}, \ldots, i_{k}\right\}$ is the set obtained by removing the integers $\mu_{1}, \mu_{1}+\mu_{2}, \ldots$ from $\{1,2, \ldots, n\}$. (For example, if $n=8$ and $\mu=(4,3,1)$, then 
$w_{\mu}=s_{1} s_{2} s_{3} s_{5} s_{6}$.) The point about choosing these representatives is that $w_{\mu}$ has minimal length in its conjugacy class, that is, we have $l\left(w_{\mu}\right) \leqslant l(w)$ for any $w \in \mathfrak{S}_{n}$ which is conjugate to $w_{\mu}$. Now, applying the defining formula for Ocneanu's trace $\tau_{z}$ to an element $w_{\mu}$ as above, we see that

$$
\tau_{z}\left(T_{w_{\mu}}\right)=z^{l\left(w_{\mu}\right)} \text { for all } \mu \vdash n .
$$

Conversely, if $\psi$ is any trace function on $\mathcal{H}_{n}(q)$ such that $\psi\left(T_{w_{\mu}}\right)=z^{l\left(w_{\mu}\right)}$ for all $\mu \vdash n$, then we necessarily have $\psi=\tau_{z}$. (This follows from the above-mentioned fact that any trace function on $\mathcal{H}_{n}(q)$ is uniquely determined by its values on the elements $T_{w_{\mu}}$.)

Now we consider the vectorspace of all trace functions on $\mathcal{H}_{n}(q)$. There is a distinguished basis of that vectorspace, constructed as follows. It is known that $\mathcal{H}_{n}(q)=\bigoplus_{\lambda \vdash n} M_{\lambda}$ where each $M_{\lambda}$ is a two-sided ideal isomorphic to a full matrix algebra over $K$. By extending the usual matrix trace on $M_{\lambda}$ to all of $\mathcal{H}_{n}(q)$ (where the extension is zero outside $M_{\lambda}$ ) we obtain a trace function $\chi_{q}^{\lambda}$ on $\mathcal{H}_{n}(q)$. The set

$$
\operatorname{Irr}\left(\mathcal{H}_{n}(q)\right)=\left\{\chi_{q}^{\lambda} \mid \lambda \vdash n\right\}
$$

is the desired basis of the space of trace functions on $\mathcal{H}_{n}(q)$. The elements of $\operatorname{Irr}\left(\mathcal{H}_{n}(q)\right)$ are called the irreducible characters of $\mathcal{H}_{n}(q)$. See [7] for a general exposition of the theory of Iwahori-Hecke algebras and their characters.

For any $z \in K$, we now have a unique expression

$$
\tau_{z}=\sum_{\lambda \vdash n} \omega_{\lambda}(q, z) \chi_{q}^{\lambda} \quad \text { with } \omega_{\lambda}(q, z) \in K,
$$

where the coefficients $\omega_{\lambda}(q, z)$ are called the weights of $\tau_{z}$.

The purpose of this note is to give a new proof of the following result, which is due to Ocneanu (unpublished; see [9]) and Wenzl [15, §3]. We shall identify a partition $\lambda \vdash n$ with its diagram, i.e., the set of all $(i, j) \in \mathbb{N} \times \mathbb{N}$ such that $1 \leqslant i \leqslant l$ and $1 \leqslant j \leqslant \lambda_{i}$, where $\lambda_{1} \geqslant \ldots \geqslant \lambda_{l}>0$ are the non-zero parts of $\lambda$. Using this convention, we denote by $c(x)=j-i$ the content and by $h(x)$ the hook length of $x \in \lambda$ (see [12, Ex. I.1.1]); furthermore, we set $n(\lambda)=\sum_{i=1}^{l}(i-1) \lambda_{i}$.

Theorem 1.1 The weights of the Ocneanu trace with parameter $z$ are given by

$$
\omega_{\lambda}(q, z)=q^{n(\lambda)} \prod_{x \in \lambda} \frac{q-1+\left(q^{c(x)}-1\right) z}{q^{h(x)}-1} .
$$

The proof we will give in Section 2 uses only some classical results about Schur polynomials and the characters of $\mathfrak{S}_{n}$ (which can be found in Macdonald's monograph [12]) and Starkey's rule (see Theorem 2.2) for the irreducible characters of $\mathcal{H}_{n}(q)$ (a self-contained proof of which can be found in [3]).

Remark 1.2 The above result has the following application. Consider the special case where $z=0$. Then $(*)$ shows that $\tau_{0}\left(T_{1}\right)=1$ and $\tau_{0}\left(T_{w_{\mu}}\right)=0$ for 
all $\mu \vdash n, \mu \neq\left(1^{n}\right)$. Thus, $\tau_{0}$ is the canonical symmetrizing trace on $\mathcal{H}_{n}(q)$ and we have

$$
\omega_{\lambda}(q, 0)=\frac{D_{\lambda}(q)}{P_{n}(q)} \quad \text { for all } \lambda \vdash n,
$$

where $P_{n}(q)=\prod_{i=1}^{n}\left(q^{i-1}+q^{i-2}+\cdots+q+1\right)$ is the Poincaré polynomial of $\mathcal{H}_{n}(q)$ and $D_{\lambda}(q)$ denotes the generic degree of $\chi_{q}^{\lambda}$; see $[7,9.4 .5]$. If we specialise $q$ to a prime power, $p^{f}$ say, then the generic degrees have a meaning in the representation theory of the general linear group $\mathrm{GL}_{n}(k)$ where $k$ is the finite field with $p^{f}$ elements. Setting $z=0$ in Theorem 1.1, we obtain the formula

$$
D_{\lambda}(q)=q^{n(\lambda)} \frac{(q-1)^{n} P_{n}(q)}{\prod_{x \in \lambda}\left(q^{h(x)}-1\right)} .
$$

This formula is originally due to Steinberg; see [7, 10.5.2] (modulo the identities concerning hook lengths in [12, I.1.1]). Thus, the weight formula for Ocneanu traces also yields a new proof for the generic degrees in type $A$. This deduction of the generic degrees from Theorem 1.1 was first described by Ram-Remmel [14].

Remark 1.3 Jones wrote in $[9$, p. 346] that there should be analogues of Ocneanu's trace for Iwahori-Hecke algebras other than those of type $A$. The trace given by Lambropoulou [10] was the first such analogue for Iwahori-Hecke algebras of type $B$. In type $B$ there are infinitely many Markov traces; these are constructed in [5] using results of [6]. Subsequently, Lambropoulou [11] constructed analogues of Ocneanu's trace for the so-called cyclotomic algebras associated with the complex reflection groups $(\mathbb{Z} / e \mathbb{Z}) \imath \mathfrak{S}_{n}$ where $e \geqslant 1$. These algebras were first defined and studied by Ariki-Koike [1] and Broué-Malle [2]. If $e=1$, one just gets the Iwahori-Hecke algebra $\mathcal{H}_{n}(q)$ associated with $\mathfrak{S}_{n}$; if $e=2$, one gets the Iwahori-Hecke algebra of type $B_{n}$.

The generalization of Ocneanu's trace to a cyclotomic algebra depends on $e$ parameters $z, y_{1}, \ldots, y_{e-1}$. The problem of determining the weights of these traces was first considered by Orellana [13]. She determined the weights for $e=2$ and for special choices of the parameters $z, y_{1}$. Then Iancu [8] found a formula (in the case $e=2$ ) which actually expresses the weights as polynomial functions in the parameters $z, y_{1}$. Furthermore, she conjectured a general weight formula for any $e \geqslant 2$. This conjecture was subsequently proved by Geck-Iancu-Malle [4]. It should be noted that both Orellana's proof and the part of the proof in [4] which is concerned with a generalization of Orellana's argument use the knowledge of the weights for Iwahori-Hecke algebras of type $A$, that is, the formula in Theorem 1.1.

Thus, the weights of Ocneanu's trace on $\mathcal{H}_{n}(q)$ play a crucial role in the determination of the weights for cyclotomic algebras. This was one of our motivations to find a new simple proof of Theorem 1.1. 


\section{Proof of Theorem 1.1}

Let $z \in K$ and let us define a trace function $\psi_{z}: \mathcal{H}_{n}(q) \rightarrow K$ by the formula

$$
\psi_{z}:=\sum_{\lambda \vdash n}\left(q^{n(\lambda)} \prod_{x \in \lambda} \frac{q-1+\left(q^{c(x)}-1\right) z}{q^{h(x)}-1}\right) \chi_{q}^{\lambda} .
$$

In order to prove Theorem 1.1, we must show that $\psi_{z}$ is the Ocneanu trace with parameter $z$. By the remarks following $(*)$, this is equivalent to showing that $\psi_{z}\left(T_{w_{\mu}}\right)=z^{l\left(w_{\mu}\right)}$ for all $\mu \vdash n$. Thus, it remains to prove the following identity:

$$
z^{l\left(w_{\mu}\right)}=\sum_{\lambda \vdash n}\left(q^{n(\lambda)} \prod_{x \in \lambda} \frac{q-1+\left(q^{c(x)}-1\right) z}{q^{h(x)}-1}\right) \chi_{q}^{\lambda}\left(T_{w_{\mu}}\right) \quad \text { for all } \mu \vdash n .
$$

For this purpose, we note that both sides can actually be expressed as polynomials in $z$ with coefficients in $K=\mathbb{Q}(q)$. Thus, if we let $\mathbf{z}$ be an indeterminate over $K$, we have to show the following identity of polynomials in $K[\mathbf{z}]$ :

$$
\mathbf{z}^{l\left(w_{\mu}\right)}=\sum_{\lambda \vdash n}\left(q^{n(\lambda)} \prod_{x \in \lambda} \frac{q-1+\left(q^{c(x)}-1\right) \mathbf{z}}{q^{h(x)}-1}\right) \chi_{q}^{\lambda}\left(T_{w_{\mu}}\right) \quad \text { for all } \mu \vdash n .
$$

In order to prove such a polynomial identity, it is enough to prove it for infinitely many specialisations of the variable $\mathbf{z}$ to elements in $K$. Following Wenzl [15], we shall use the specialisations

$$
\mathbf{z} \mapsto z_{r}:=q^{r} \frac{1-q}{1-q^{r}} \quad \text { for all } r \in \mathbb{N}, r \geqslant n .
$$

The point about this choice is that we have the following identity:

$$
q^{n(\lambda)} \prod_{x \in \lambda} \frac{q-1+\left(q^{c(x)}-1\right) z_{r}}{q^{h(x)}-1}=\left(\frac{1-q}{1-q^{r}}\right)^{n} s_{\lambda}\left(1, q, q^{2}, \ldots, q^{r-1}\right),
$$

where $s_{\lambda}$ is the Schur polynomial corresponding to $\lambda$ in $r$ variables $x_{1}, \ldots, x_{r}$; see [12, Ex. I.3.1]. Hence we must show the following identity for $\mu \vdash n, r \geqslant n$ :

$$
q^{r l\left(w_{\mu}\right)}\left(\frac{1-q^{r}}{1-q}\right)^{l(\mu)}=\sum_{\lambda \vdash n} s_{\lambda}\left(1, q, q^{2}, \ldots, q^{r-1}\right) \chi_{q}^{\lambda}\left(T_{w_{\mu}}\right) .
$$

In order the evaluate the right hand side, we use two basic results about the characters of $\mathfrak{S}_{n}$ and $\mathcal{H}_{n}(q)$ : Frobenius' character formula and Starkey's rule. Denote by $\operatorname{Irr}\left(\mathfrak{S}_{n}\right)=\left\{\chi^{\lambda} \mid \lambda \vdash n\right\}$ the set of irreducible characters of $\mathfrak{S}_{n}$.

Theorem 2.1 (Frobenius' character formula; see [12, I.7]) For any $r \geqslant$ $n$ and any partition $\nu \vdash n$, we have

$$
\sum_{\lambda \vdash n} s_{\lambda}\left(x_{1}, \ldots, x_{r}\right) \chi^{\lambda}\left(w_{\nu}\right)=\prod_{i \geqslant 1}\left(x_{1}^{\nu_{i}}+\cdots+x_{r}^{\nu_{i}}\right),
$$

where $\nu_{1}, \nu_{2}, \ldots$ are the non-zero parts of $\nu$. 
The following rule shows how $\chi_{q}^{\lambda}$ is determined by $\chi^{\lambda}$. First note that the labellings are compatible in the following sense. We have $\chi_{q}^{\lambda}\left(T_{w}\right) \in \mathbb{Q}[q]$ for $\lambda \vdash n$ and $w \in \mathfrak{S}_{n}$. Then, by Tits' deformation theorem (see $[7,8.1 .7]$ ), we have

$$
\chi^{\lambda}(w)=\left.\chi_{q}^{\lambda}\left(T_{w}\right)\right|_{q=1} \quad \text { for all } \lambda \vdash n \text { and } w \in \mathfrak{S}_{n} .
$$

Theorem 2.2 (Starkey's rule; see [3]) For partitions $\mu, \nu \vdash n$, we define

$$
p_{\mu}^{\nu}(q):=\frac{\left|C_{\nu} \cap \mathfrak{S}_{\mu}\right|}{\left|\mathfrak{S}_{\mu}\right|}(q-1)^{-l(\mu)} \prod_{i \geqslant 1}\left(q^{\nu_{i}}-1\right),
$$

where $\nu_{1}, \nu_{2}, \ldots$ are the non-zero parts of $\nu$ and $C_{\nu}$ denotes the conjugacy class of elements of cycle type $\nu$ in $\mathfrak{S}_{n}$. Then we have

$$
\chi_{q}^{\lambda}\left(T_{w_{\mu}}\right)=\sum_{\nu \vdash n} p_{\mu}^{\nu}(q) \chi^{\lambda}\left(w_{\nu}\right) \quad \text { for all } \lambda, \mu \vdash n .
$$

Using the above two results, we can now evaluate the right hand side of (3).

$$
\begin{aligned}
\text { r.h.s. of }(3) & =\sum_{\lambda \vdash n} s_{\lambda}\left(1, q, q^{2}, \ldots, q^{r-1}\right) \sum_{\nu \vdash n} p_{\mu}^{\nu}(q) \chi^{\lambda}\left(w_{\nu}\right) \quad \text { by Theorem } 2.2 \\
& =\sum_{\nu \vdash n} p_{\mu}^{\nu}(q) \sum_{\lambda \vdash n} s_{\lambda}\left(1, q, q^{2}, \ldots, q^{r-1}\right) \chi^{\lambda}\left(w_{\nu}\right) \\
& =\sum_{\nu \vdash n} p_{\mu}^{\nu}(q) \prod_{i \geqslant 1}\left(1+q^{\nu_{i}}+q^{2 \nu_{i}}+\cdots+q^{(r-1) \nu_{i}}\right) \quad \text { by Theorem } 2.1 \\
& =\sum_{\nu \vdash n} p_{\mu}^{\nu}(q) \prod_{i \geqslant 1} \frac{q^{r \nu_{i}}-1}{q^{\nu_{i}}-1}=\left(\frac{1-q^{r}}{1-q}\right)^{l(\mu)} \sum_{\nu \vdash n} p_{\mu}^{\nu}\left(q^{r}\right)
\end{aligned}
$$

where the last equality is proved by using the defining formula for $p_{\mu}^{\nu}(q)$ and applying it to $p_{\mu}^{\nu}\left(q^{r}\right)$ as well. Thus, it remains to show the following identity:

$$
q^{r l\left(w_{\mu}\right)}=\sum_{\nu \vdash n} p_{\mu}^{\nu}\left(q^{r}\right) \quad \text { for all } \mu \vdash n \text { and } r \geqslant n \text {. }
$$

For this purpose, we consider the 1-dimensional representation $\operatorname{ind}_{q}: \mathcal{H}_{n}(q) \rightarrow$ $K$ given by $\operatorname{ind}_{q}\left(T_{w}\right)=q^{l(w)}$. Specialising $q \mapsto 1$ shows that ind $q$ corresponds to the trivial character of $\mathfrak{S}_{n}$. Hence, by Theorem 2.2, we have

$$
\sum_{\nu \vdash n} p_{\mu}^{\nu}(q)=\operatorname{ind}_{q}\left(T_{w_{\mu}}\right)=q^{l\left(w_{\mu}\right)} .
$$

We can do the same with $q^{r}$ instead of $q$ and, thus, obtain (4). This completes the proof of Theorem 1.1.

\section{References}

[1] S. Ariki And K. KoIKE, A Hecke algebra of $(\mathbb{Z} / r \mathbb{Z})$ ¿ $\mathfrak{S}_{n}$ and construction of its irreducible representations, Adv. Math. 106 (1994), 216-243. 
[2] M. Broué And G. Malle, Zyklotomische Heckealgebren. Astérisque 212 (1993), 119-189.

[3] M. GECK, The character table of the Iwahori-Hecke algebra of the symmetric group : Starkey's Rule. C. R. Acad. Sci. Paris Sér I 329 (1999), $361-366$.

[4] M. Geck, L. Iancu and G. Malle, Weights of Markov traces and generic degrees. Indag. Mathem., N. S., 11 (2000), 379-397.

[5] M. Geck and S. Lambropoulou, Markov traces and knot invariants related to Iwahori-Hecke algebras of type $B$. J. reine angew. Math. 482 (1997), 191-213.

[6] M. Geck and G. Pfeiffer, On the irreducible characters of Hecke algebras, Adv. Math. 102 (1993), 79-94.

[7] M. Geck and G. Pfeiffer, Characters of finite Coxeter groups and Iwahori-Hecke algebras, London Math. Soc. Monographs, New Series 21, Oxford University Press, New York, 2000.

[8] L. IANCU, Markov traces and generic degrees in type $B_{n}$, J. of Algebra 236 (2001), 731-744.

[9] V. F. R. Jones, Hecke algebra representations of braid groups and link polynomials, Ann. Math. 126 (1987), 335-388.

[10] S. Lambropoulou, Solid torus links and Hecke algebras of $B$-type, Proceedings of the Conference on Quantum Topology (Manhattan, KS, 1993), 225-245, World Sci. Publishing, River Edge, NJ, 1994.

[11] S. Lambropoulou, Knot theory related to generalized and cyclotomic Hecke algebras of type $B$, J. Knot Theory Ramifications 8 (1999), 621658 .

[12] I. G. Macdonald, Symmetric functions and Hall polynomials, Second edition, Oxford Math. Monographs, Clarendon Press, Oxford, 1995.

[13] R. C. Orellana, Weights of Markov traces on Hecke algebras, J. reine angew. Math. 508 (1999), 157-178.

[14] A. Ram and J. B. Remmel, Applications of the Frobenius formulas for the characters of the symmetric group and the Hecke algebras of type $A$, J. Algebraic Combin. 6 (1997), 59-87.

[15] H. WenzL, Hecke algebras of type $A_{n}$ and subfactors, Invent. Math. 92 (1988), 349-383.

Institut Girard Desargues, bat. Jean Braconnier, Université Lyon 1, 21 aV Claude Bernard, F-69622 Villeurbanne cedex, France

E-mail addresses: geck@desargues.univ-lyon1.fr, jacon@desargues.univ-lyon1.fr 